\title{
VHEeP: A very high energy electron-proton collider based on proton-driven plasma wakefield acceleration
}

\author{
A. Caldwell \\ Max Planck Institute for Physics, Munich, Germany \\ E-mail: caldwell@mpp.mpg.de \\ M. Wing ${ }^{* \dagger}$ \\ UCL, London, UK \\ E-mail: m.wing@ucl.ac.uk
}

\begin{abstract}
Based on current CERN infrastructure, an electron-proton collider is proposed at a centre-ofmass energy of about $9 \mathrm{TeV}$. A $7 \mathrm{TeV}$ LHC bunch is used as the proton driver to create a plasma wakefield which then accelerates electrons to $3 \mathrm{TeV}$, these then colliding with the other $7 \mathrm{TeV}$ LHC proton beam. The basic parameters of the collider are presented, which although of very high energy, has integrated luminosities of the order of $1 \mathrm{pb}^{-1} /$ year. For such a collider, with a centre-of-mass energy 30 times greater than HERA, parton momentum fractions, $x$, down to about $10^{-8}$ are accessible for $Q^{2}$ of $1 \mathrm{GeV}^{2}$ and could lead to effects of saturation or some other breakdown of DGLAP being observed. The total photon-proton cross section can be measured up to very high energies and also at different energies as the possibility of varying the electron beam energy is assumed; this could have synergy with cosmic-ray physics. Other physics which can be pursued at such a collider are contact interaction searches, such as quark and electron substructure, and measurements of the proton structure as well as other more conventional measurements of QCD at high energies and in a new kinematic regime. The events at very low $x$ will lead to electrons and the hadronic final state produced at very low angles and so a novel spectrometer device will be needed to measure these. First ideas of the physics programme of such a collider are given.
\end{abstract}

XXIII International Workshop on Deep-Inelastic Scattering

27 April - May 12015

Dallas, Texas

\footnotetext{
* Speaker.

${ }^{\dagger}$ Also at Universität Hamburg and supported by DESY and the Alexander von Humboldt Foundation.
} 


\section{Introduction}

The HERA $e P$ machine is so far the only lepton-hadron collider worldwide. Due to its centreof-mass energy of about $300 \mathrm{GeV}$, HERA dramatically extended the kinematic reach for the deep inelastic scattering process compared to previous fixed-target experiments [1]. A broad range of physics processes and new insights were gleaned from HERA which complemented the $p \bar{p}$ and $e^{+} e^{-}$colliders, the Tevatron and LEP. The LHeC project [2] is a proposed $e P$ collider with significantly higher energy and luminosity than HERA with a programme to investigate Higgs physics and QCD, to search for new physics, etc.. This will use significant parts of the LHC infrastructure at CERN with different configurations, such as $e A$, also possible. These proceedings consider the possibility of having a very high energy electron-proton collider (VHEeP) with an $e P$ centre-ofmass energy of about $9 \mathrm{TeV}$, a factor of six higher than proposed for the $\mathrm{LHeC}$ and a factor of 30 higher than HERA.

The VHEeP machine would strongly rely on the use of the LHC beams and the technique of plasma wakefield acceleration to accelerate electrons to $3 \mathrm{TeV}$ over relatively short distances. Given such an acceleration scheme, the luminosities will be relatively low, $\mathscr{O}\left(\mathrm{pb}^{-1}\right)$. Given such an increase in centre-of-mass energy, the VHEeP collider will probe a new regime in deep inelastic scattering, particularly at low values of the probed fractional parton momentum, $x$. These proceedings will look at first investigations of the physics potential of such a collider as well as first ideas of its technical implementation.

\section{The VHEeP accelerator and detector}

Given the limitation in the accelerating gradient for RF cavities of about $100 \mathrm{MV} / \mathrm{m}$, due to structural breakdown, a future collider with electron energies at the $\mathrm{TeV}$ scale requires an accelerator of lengths 10s of kilometres. Using a novel technique, plasma wakefield acceleration [3], gradients up to about $100 \mathrm{GV} / \mathrm{m}$ have been observed [4], although there are many challenges to be overcome before this scheme can be used in a real accelerator. Plasma wakefield acceleration overcomes the limitation on the accelerating gradient as the plasma is already ionised itself. The phenomenon of plasma wakefield acceleration relies on a compact particle or laser beam to drive the process in plasma. In the case of a proton beam, the free plasma electrons are attracted to the incoming beam, overshoot and create regions of high positive charge density due to the ions which have remained static during this process. The plasma electrons are attracted to these regions of high positive charge and so an oscillating motion of the electrons is set up in which a strong accelerating gradient is initiated in the direction of the proton beam. Given the high energy of existing proton beams, they could accelerate a witness bunch of electrons injected into the wakefield to the $\mathrm{TeV}$ scale [5]. Proton-driven plasma wakefield acceleration has so far not been demonstrated experimentally and a collaboration, AWAKE, has been formed to show this using the CERN SPS beam [6]. The aim is to start taking data in 2016 and, in an initial 2-year running period, demonstrate $\mathscr{O}(\mathrm{GeV})$ acceleration of electrons within $10 \mathrm{~m}$ of plasma.

Simulations have shown that using the $7 \mathrm{TeV}$ LHC proton beam as designed, electrons can be accelerated to $3 \mathrm{TeV}$ in $4 \mathrm{~km}$ [7], about a factor of 10 less than needed for conventional RF acceleration. For the VHEeP collider, such an electron beam is then assumed to collide with the 
other $7 \mathrm{TeV}$ LHC proton beam, giving an $e P$ centre-of-mass energy of about $9 \mathrm{TeV}$. This is shown schematically in figure 1(a). The emphasis is on using current CERN infrastructure where possible, i.e. the LHC with minimum modifications. To obtain a new beamline within the LHC ring, highgradient magnets will be needed to provide a smaller bending radius. It is also assumed that the electron beam energy can be varied within the constraints of the length of the accelerator structure and so varying up to the maximum possible is assumed; this is motivated by the possibility to measure extra physics processes.

(a)

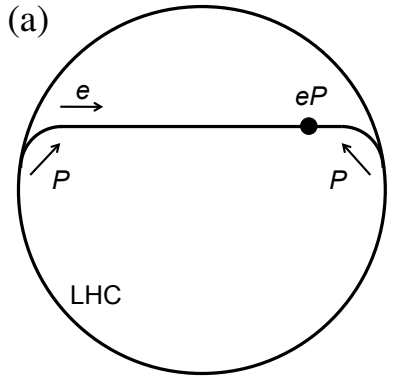

(b)

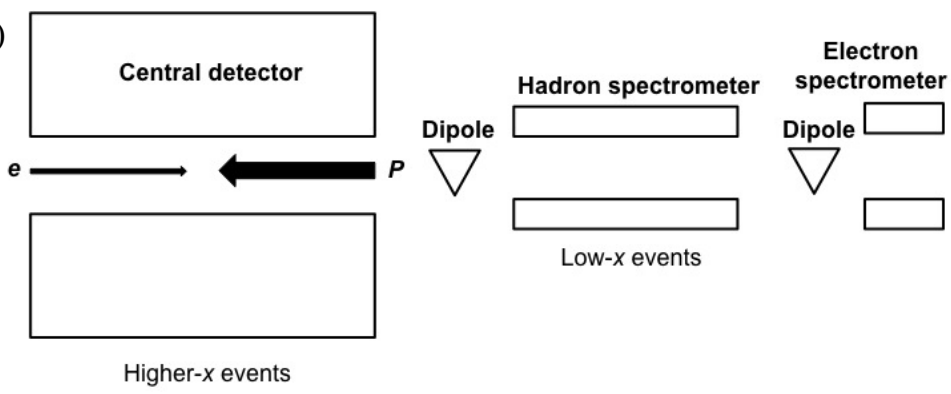

Figure 1: (a) Sketch of VHEeP collider. One of the proton beams from the LHC is used as a driver to accelerator electrons which subsequently collide with the other LHC proton beam. The $e P$ interaction point is indicated. (b) Sketch of VHEeP detector showing the need for a central, conventional collider detector and a long arm of spectrometer detectors to measure the scattered electrons and hadronic final state at low $x$.

Although the energy of VHEeP will be very high, obtaining high luminosities will be, as with all plasma wakefield acceleration schemes, a challenge. The luminosity is determined using the standard formula for a collider,

$$
\mathscr{L}=\frac{f \cdot N_{e} \cdot N_{P}}{4 \pi \sigma_{x} \cdot \sigma_{y}} .
$$

It is assumed that about 3000 bunches are delivered every 30 minutes, giving a frequency, $f$, of $2 \mathrm{~Hz}$. The number of protons, $N_{P}$, and number of electrons, $N_{e}$, per bunch are $4 \times 10^{11}$ and $1 \times$ $10^{11}$, respectively. Assuming a beam cross section, $\sigma_{x} \sim \sigma_{y}$, of $4 \mu \mathrm{m}$, gives a luminosity of $4 \times$ $10^{28} \mathrm{~cm}^{-2} \mathrm{~s}^{-1}$. Running the collider for a large fraction of the year gives an integrated luminosity of about $1 \mathrm{pb}^{-1} /$ year.

Therefore, the expected luminosities are lower than were achieved at HERA and significantly lower than proposed for the LHeC. Hence, for the VHEeP facility, a physics case needs to be made for $e P$ physics at very high energies, but moderate luminosities and is the initial focus of the subsequent sections in these proceedings and will be for further studies of the VHEeP accelerator.

\section{Physics at VHEeP}

An initial list of some of the possible areas of investigation for a high energy $e P$ collider with moderate luminosities are:

- Measurements of cross sections at very low $x$ and investigation of saturation; 
- Investigation of contact interactions, e.g. radius of quarks and electrons;

- Measurements of the total $\gamma P$ cross section at high energies and at many different energies. This will allow a precise determination of its energy dependence and its relation to cosmicray physics can be investigated;

- Investigation of the structure of the proton and photon, in particular the longitudinal proton structure function, $F_{L}$, which will also profit from the possibility to change the beam energy as well as results from $e A$ scattering;

- Further tests of QCD such as extraction of the strong coupling at high energies.

In these proceedings, the first is investigated in more detail, whilst the others will be pursued in the future. This list is not exhaustive and there may be other interesting possibilities.

In order to investigate the results of $e P$ collisions at a centre-of-mass energy of $9 \mathrm{TeV}$, the ARIADNE 4.12 Monte Carlo programme was used to generate events over a wide kinematic region. The exchanged boson virtuality, $Q^{2}$, was required to be above $1 \mathrm{GeV}^{2}$, the squared photon-proton centre of mass energy, $W^{2}$, was required to be above $5 \mathrm{GeV}^{2}$ and the fraction of the proton's momentum carried by the struck quark, $x$, was required to be greater than $10^{-7}$. The requirement on $x$ was due to technical issues running the generator at such low $x$ and will be relaxed in the future. A test sample with an integrated luminosity of about $0.01 \mathrm{pb}^{-1}$ was generated. Investigation of the scattered electron showed that a kinematic peak at $3 \mathrm{TeV}$ is observed with the electron scattered at very low angles, essentially along the beamline axis. This then requires detectors at shallow angles along the beam to measure the scattered electron. The hadronic final state covered all polar angles and so a conventional colliding-beam detector will be needed. However, at low $x$, the hadronic final state is produced at low angles and the angle is essentially zero and along the beamline for $x<10^{-6}$. This again requires instrumentation along the beamline. A sketch of a detector system is shown in figure 1(b), where a series of spectrometers are needed in which the hadrons or electrons are bent away from the beamline and measured in dedicated detectors.

Distributions of the kinematic variables describing deep inelastic scattering, $x, Q^{2}$ and the inelasticity, $y$, are shown in figure 2 along with the correlation of $Q^{2}$ and $x$. As expected, the distributions peak at low values of $x, Q^{2}$ and $y$, with a strong correlation between $x$ and $Q^{2}$, also showing that once the technical Monte Carlo cut on $x$ is removed, values of $x \sim 10^{-8}$ for $Q^{2}>$ $1 \mathrm{GeV}^{2}$ are possible.

\section{The photon-proton cross section at low $x$}

With such extended reach in $x, \mathrm{VHEeP}$ can be used to investigate the nature of the proton at very low parton momentum fractions, investigating the onset of saturation or other phenomena not described by standard QCD evolution of the parton densities. A complementary approach is to consider the cross sections as a function of $1 / x$ or the coherence length, $l$. The coherence length is the distance over which a quark-antiquark pair can survive having come from a photon radiated by the incoming electron. If the cross sections become the same as a function of $Q^{2}$, the photon states have had enough time to evolve into a universal size. 

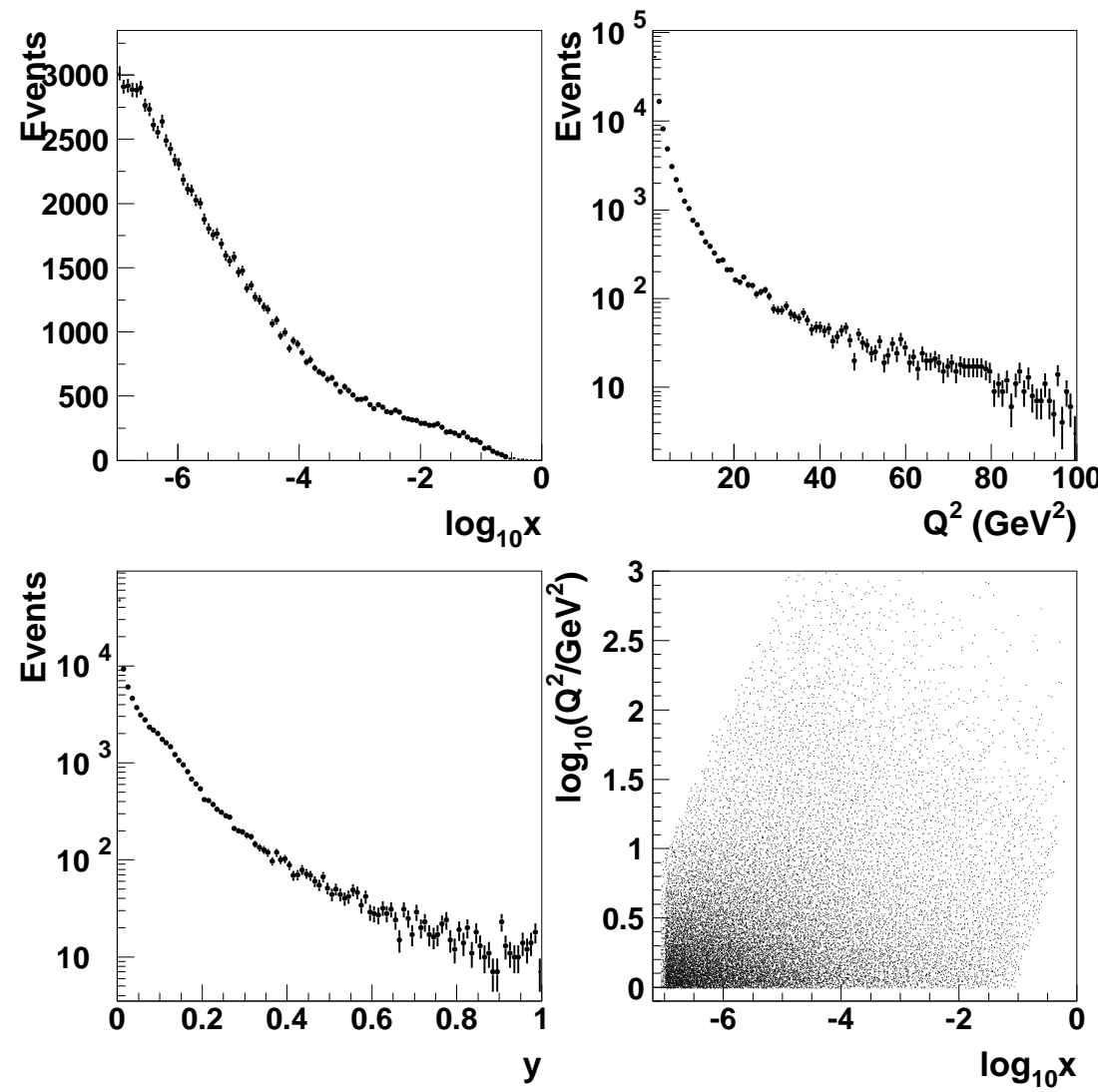

Figure 2: Kinematic variables, $x, Q^{2}$ and $y$, and the correlation between $Q^{2}$ and $x$ generated with the ARIDANE Monte Carlo generator. For technical reasons, the events were limited to have an $x$ value above $10^{-7}$. The number of events is plotted for a luminosity of about $0.01 \mathrm{pb}^{-1}$.

Initially the photon-proton cross section, $\sigma^{\gamma P}$, versus $l$, according to the definition by Stodolsky [8] $\left(l=\hbar c /\left(2\langle x\rangle M_{P}\right)\right)$, is shown for the HERA data [1] in figure 3. The data extend up to $l \sim 3 \times 10^{4} \mathrm{fm}$ which corresponds to $x \sim 3.5 \times 10^{-6}$. The data are well described by a fit for each $Q^{2}$ value using the simple parametrisation $\sigma_{0} l^{\lambda}$. The figure also shows that the fits are converging for increasing $l$ and that it is expected that the cross section becomes independent of $Q^{2}$ at large $l$ (or small $x$ ).

Figure 4 shows the fits for selected $Q^{2}$ values extrapolated to higher $l$, where the fits cross at a value of $l \sim 3 \times 10^{8} \mathrm{fm}$ or $x \sim 3.5 \times 10^{-10}$. An indication of the largest $l$ values that can be measured at VHEeP for each $Q$ value is also shown. This demonstrates that measurements at $Q^{2}<1 \mathrm{GeV}^{2}$ will be very important in order to measure the region where the cross sections are expected to unify. However, in the region $1<Q^{2}<10 \mathrm{GeV}^{2}$, a very large number of events will be collected, even with the relatively low luminosities, and so able to constrain the fits for $l>10^{6} \mathrm{fm}$. At higher $Q^{2}$, there will also be significant amounts of data, also at higher $l$ than was measured at HERA, and so will significantly constrain the extrapolation of the fits to higher $l$. Further and more quantitative studies are needed, but already these initial investigations show that VHEeP will 


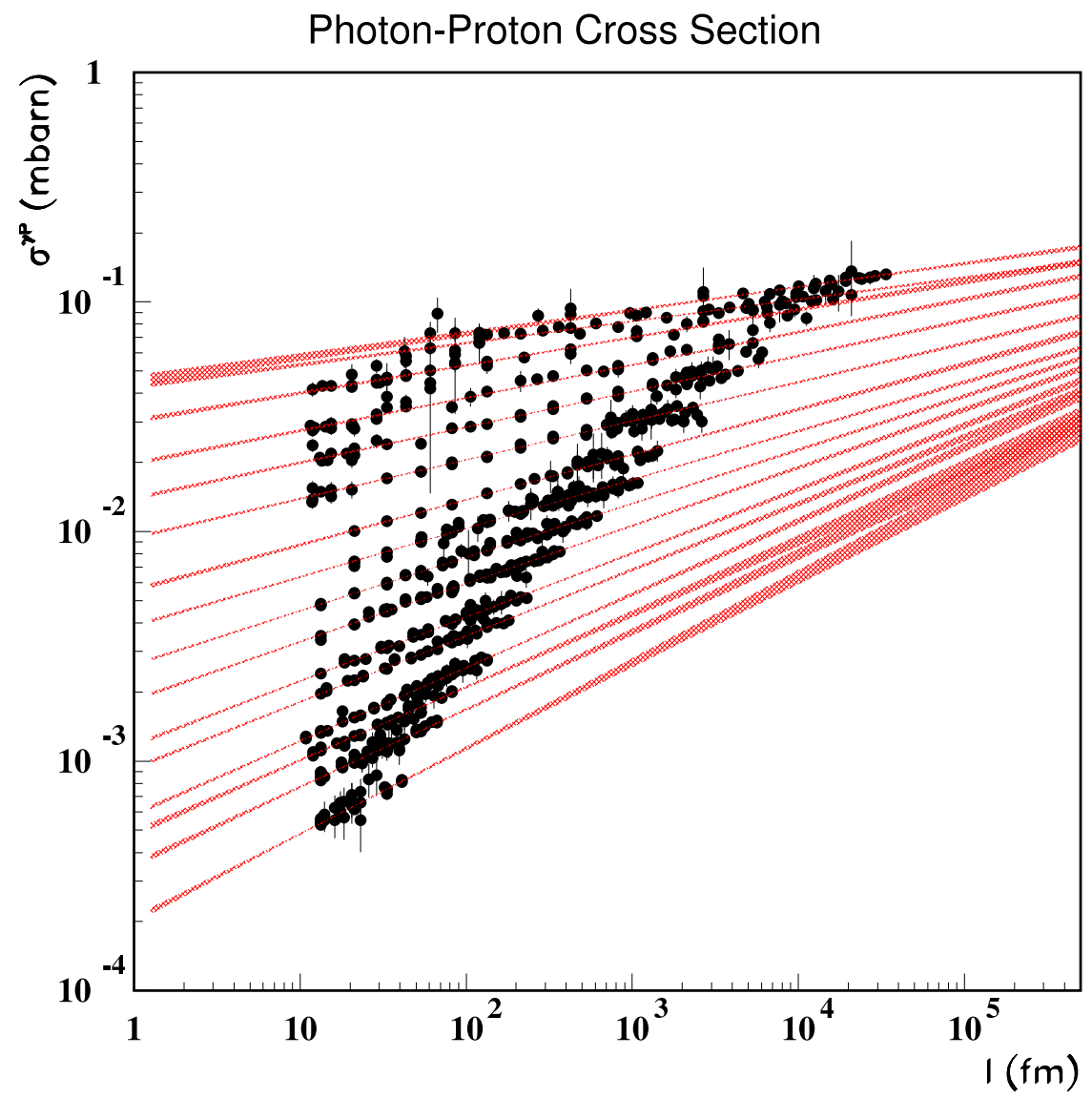

Figure 3: The photon-proton cross section, $\sigma^{\gamma P}$, shown versus the coherence length, $l$, for HERA data [1] at fixed values of $Q^{2}$ in the range 0.25 (top points) to $200 \mathrm{GeV}^{2}$ (bottom points). The data are fit for each $Q^{2}$ value with a function $\sigma_{0} l^{\lambda}$.

be able to constrain the onset of saturation in the proton.

\section{Summary and outlook}

In these proceedings, an idea for a very high energy electron-proton collider, VHEeP, has been presented. Although of modest luminosities, $1 \mathrm{pb}^{-1} /$ year, the centre-of-mass energy of $9 \mathrm{TeV}$ opens up a whole new kinematic region in $e P$ collisions. Initial studies already indicate that effects such as saturation in the proton could be observed. More work on developing a broad physics programme for VHEeP is ongoing.

\section{Acknowledgements}

L Lönnblad is gratefully acknowledged for discussions using the ARIADNE Monte Carlo programme in this new kinematic regime. E. Shaposhnikova is also gratefully acknowledged for discussions on the LHC beam parameters. 


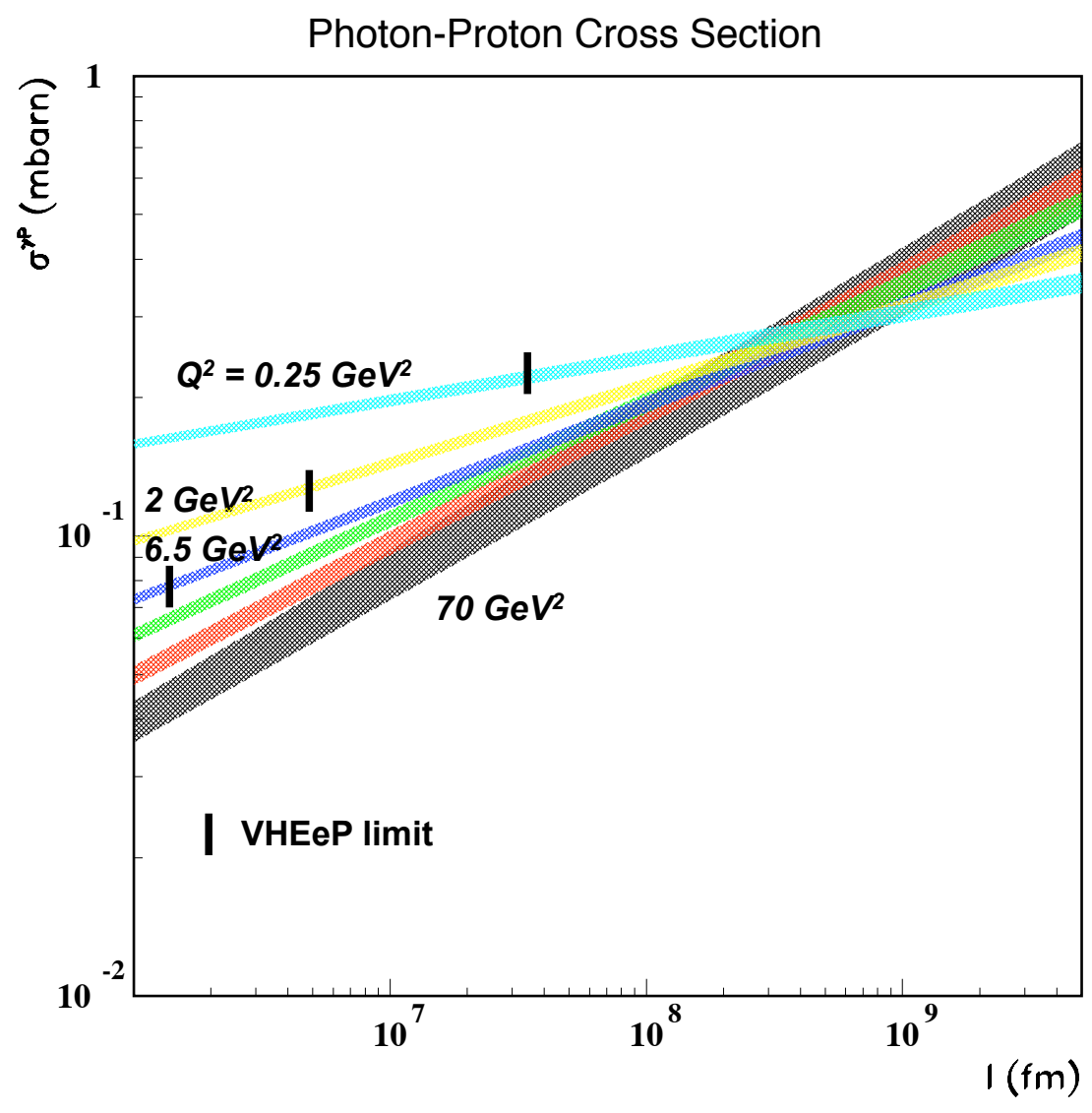

Figure 4: Fits of $\sigma_{0} l^{\lambda}$ extrapolated with uncertainties to high $l$ (low $x$ ) for selected $Q^{2}$ values. The upper kinematic limit of where VHEeP measurements will end is indicated (black vertical lines).

\section{References}

[1] H. Abramowicz et al., H1 and ZEUS Collaborations, arXiv:1506.06042.

[2] P. Newman and A. Stasto, Nature Phys. 9 (2013 448;

LHeC Study Group, J.L. Abelleira Fernandez et al., J. Phys. G 39 (2012) 075001.

[3] T. Tajima and J.M. Dawson, Phys. Rev. Lett. 43 (1979) 267.

[4] W.P. Leemans et al., Nature Phys. 2 (2006) 696;

I. Blumenfeld et al., Nature 445 (2007) 741.

[5] A. Caldwell et al., Nature Phys. 5 (2009) 363.

[6] AWAKE Collaboration, R. Assmann et al., Plasma Phys. Control. Fusion 56 (2014) 084013 ; AWAKE Collaboration, Design Report, CERN-SPSC-2013-013.

[7] A. Caldwell and K. Lotov, Phys. Plasmas 18 (2011) 103101.

[8] L. Stodolsky, Phys. Lett. B 325 (1994) 505. 